Jean Léo Léonard

Ksenija Djordjevic Léonard

Université Paul-Valéry Montpellier 3

EA-739 DIPRALANG

France

ksenija.leonard@univ-montp3.fr

jean.leonard@univ-montp3.fr
UDK 81'27:811.163.6'28(450.36)

DOI: $10.4312 /$ vestnik.13.261-279

\title{
UN REGARD SOCIOLINGUISTIQUE SUR LE RESIANO
}

\section{INTRODUCTION}

La présente contribution est conçue comme un hommage à notre ami et collègue Gregor Perko, linguiste et professeur de français à l'Université de Ljubljana. En tant que linguiste, il a collaboré avec Jean Léo Léonard, notamment dans le cadre d'une recherche sur la conjugaison slovène. Ce dernier l'avait convié en qualité de professeur invité à enseigner durant trois mois à Sorbonne Université, de mars à mai 2019. Ils avaient organisé tous deux une journée d'études sur la modélisation diasystémique, que Jean Léo Léonard avait d'ailleurs dédiée à Gregor Perko - qui en avait été le premier surpris, et avait protesté amicalement, avec toute la modestie dont il était capable, qui en faisait un compagnon de travail si convivial. En tant que professeur de français, il était pressenti pour participer à un ouvrage collectif sur l'apprentissage du français dans les Balkans co-dirigé par Ksenija Djordjevic Léonard.

Fin connaisseur des dialectes slovènes, Gregor n'a jamais eu l'occasion de travailler sur le résian de manière approfondie, avant l'article rédigé en collaboration avec Jean Léo Léonard pour la revue Verbum. Mais tous deux en rêvaient, et évoquaient souvent la question, tant les données de la flexion verbale de cette variété apportaient des lumières inespérées sur le fonctionnement du diasystème slovène, en tant que pièce du puzzle sans laquelle l'image d'ensemble resterait incomplète. La présente contribution s'inscrit donc dans la continuité de cette réflexion, et prolonge ce premier travail, en y ajoutant la dimension sociolinguistique - celle restée inaccessible aux deux compères. Ainsi, nous proposons de porter ici un regard externe sur le résian à partir d'une sélection de sources bibliographiques, de nos observations de terrain d'août 2021, mais aussi et surtout en mobilisant nos connaissances de sociolinguistes ayant déjà travaillé sur la diversité linguistique de la zone transfrontalière italo-slovène. Enfin, cette publication comblera un manque : si les linguistes et sociolinguistes slovènes et italiens n'auront que 
peu de choses à apprendre de ces lignes, le lecteur francophone découvrira tout un monde inconnu, caché à l'ombre du Mont Kanin.

Mais il y a plus que cela. La vallée de Resia représente un cas original d'expression d'une identité fluctuante et ambivalente : si la variété linguistique qui subsiste, et devrions-nous dire résiste, dans les villages de la vallée appartient au système linguistique slovène et constitue un de ses dialectes du centre-ouest, au sein du continuum linguistique de cette langue (Léonard et Perko 2020) - la dialectologie slovène le traite comme un dialecte du groupe du littoral (primorska narečna skupina) -, les habitants, quant à eux, ne se sentent pas tous Slovènes, ou du moins ne se déclarent pas comme tels. En outre, l'aménagement linguistique associatif ( « de par en bas ») qui se réalise dans cette micro-région « italo-slovène » des confins du Frioul, présente bien des aspects d'exemplarité, du point de vue de la revitalisation des « langues (et dialectes) en danger », comme nous allons le suggérer au terme de ce survol.

\section{1}

\section{UNE IDENTITÉ AMBIVALENTE}

Le Val Resia est une vallée d'une vingtaine de kilomètres de la région Frioul-Vénétie Julienne, au nord de l'Italie. Elle porte le nom de la rivière qui la traverse, et côtoie la frontière slovène. Ses principaux villages ou hameaux sont San Giorgio, Prato, Gniva, Oseacco et Stolvizza en italien (respectivement Bilä, Ravanzä, Njïwa, Osoanë et Solbiza, en résian, selon la signalisation routière locale). Peuplée de longue date par les populations slaves, en raison de son enclavement et du relief qui la caractérise, la vallée a maintenu ses particularités et ses distances avec la langue slovène voisine, qui a suivi son évolution dans un contexte différent, avant de s'imposer comme la langue nationale en Slovénie.

Ce sentiment de singularité qui caractérise le Val Resia fait que quelques-uns de ses fervents militants réclament une reconnaissance «à part » auprès de l'État italien, qui les considère comme partie intégrante de la minorité slovène, par ailleurs reconnue, y compris dans la loi $n^{\circ} 482$ de $1999^{1}$, ainsi que dans la loi nationale 38/2001, et la loi régionale 26/2007. Mais cette auto-identification très forte comme un groupe ethnolinguistique $\grave{a}$ part irait plus loin : certains se déclarent davantage proches des Slaves orientaux (ex. Russes) que des Slaves méridionaux que sont les Slovènes. Ernst Steinicke et al. précisent, en 2011, à ce sujet :

1 On peut ainsi lire dans l'article 2 : «En application de l'article 6 de la Constitution et en harmonie avec les principes généraux établis par les organisations européennes et internationales, la République protège la langue et la culture des populations albanaise, catalane, germanique, grecque, slovène et croate et celles de langue française, francoprovençale, frioulane, ladine, occitane et sarde ». [In attuazione dell'articolo 6 della Costituzione e in armonia con i princípi generali stabiliti dagli organismi europei e internazionali, la Repubblica tutela la lingua e la cultura delle popolazioni albanesi, catalane, germaniche, greche, slovene e croate e di quelle parlanti il francese, il franco-provenzale, il friulano, il ladino, l'occitano e il sardo ». Cf. https://www.camera.it/parlam/leggi/994821.htm. 
Par conséquent, une bonne partie des habitants slovènes autochtones de Resia ne veut pas être comptabilisée avec les Slovènes et n'identifie pas les dialectes villageois archaïques comme apparentés à la langue slovène. Les habitants tendent à exprimer leur identification locale par leur relation à leurs villages respectifs plutôt que par une perception d'eux-mêmes qui les lie à la culture slovène. En outre, dans ses enquêtes auprès d'experts, Steinicke (1991) a découvert que certains résidents de Resia - en termes d'ethnicité - se sentent même russes ! Il ne fait pas de doute que cette perception de soi-même n'apporte aucune aide à la préservation de cette petite minorité à l'époque de la mondialisation. (Steinicke et al. 2011).

Le rapport au territoire, au village ou à la vallée est ici, visiblement, ce qui exprime le mieux l'identité collective de la population. Quant à la langue, certaines hésitations, dans le passé, sur les origines des habitants slaves de la vallée ont laissé des traces. On peut penser ici au travail du linguiste polonais Jan Baudouin de Courtenay qui a, parmi les premiers, dans la deuxième moitié du XIXe siècle, exprimé cette hypothèse de proximité avec le russe avant de relativiser plus tard ses propos ${ }^{2}$. Dans son travail richement documenté sur les différentes hypothèses qui défendaient les origines « exotiques » des Résians, Roberto Dapit (2001) évoque, outre Baudouin de Courtenay, le témoignage du célèbre écrivain polonais Jan Potocki de 1790, qui rapporte que les habitants lui avaient soutenu être différents des Slaves de la Carinthie, ou encore du philologue russe Izmail Sreznevskij qui a noté sur place, en 1841, un fait bien curieux : " presque personne à Resia n'a jamais entendu parler de la Russie, mais beaucoup écoutent avec amour les accents russes $»^{3}$ (cité par Dapit $2001: 303$ ). Roberto Dapit précise que si ces hypothèses, mythes et légendes ont pu trouver et trouvent encore un auditoire, c'est parce qu'il se trouve des Résians pour défendre ces construits : «Il existe de nombreux témoignages recueillis au fil du temps qui attribuent aux Résians une origine " exotique », de nature même étiologique, dans le sens où les Résians eux-mêmes répandent des idées similaires sur leurs origines $»^{4}(2001: 301)$. Il ajoute :

Que de telles croyances aient pu fasciner dans les époques passées n'est pas surprenant, mais l'aspect le plus intéressant réside dans le fait qu'encore aujourd'hui les mêmes idées sont embrassées pour affirmer une identité et en

2 Avant même l'arrivée à Resia de Jan Baudouin de Courtenay, un vicaire d'origine résiane, Stefano Valente, s'est opposé à l'« hypothèse russe » : «Donc d'après ces données aussi on peut et on doit retenir que la variété parlée à Resia est fondamentalement un dialecte du slave carniol et carinthien, et non serbe, tchèque et encore moins - russe » [Dunque anche da questi dati si può e si deve ritenere che il linguaggio parlato in Resia in sostanza sia un dialetto dello Slavo Cragnolino e Carinziano, e non mai Serbo, né Czeko, e tanto meno - Russo.] (cité par Dapit 2001 : 303). $3[[\ldots]$ quasi nessuno a Resia ha mai sentito parlare della Russia, ma molti con amore nativo ascoltano gli accenti russi.]

4 [Le testimonianze raccolte nel tempo che attribuiscono ai resiani un'origine « esotica » sono numerose, anche di natura eziologica, nel senso che gli stessi resiani diffondono simili idee sulle proprie origini.] 
rejeter une autre, la slovène. En effet, la majeure partie de la population résiane refuse l'appartenance ethnique au peuple slovène dont elle est historiquement toujours restée, sauf pendant de courtes périodes, divisée par une frontière plus ou moins impénétrable. ${ }^{5}(2001: 305)$.

A cela s'ajoutent, dans l'imaginaire collectif local, les hypothèses sur une proximité plus importante avec le croate, le tchèque ou le polonais... toujours dans l'idée de marquer la différence avec le slovène. Cette tendance a été favorisée encore davantage par la non présence du slovène standard dans la vie locale du Val Resia, qui ne le reconnaît pas comme sa langue-toit (Toso, 2008 : 179), à la différence de l'opinion répandue dans les communes plus proches de la frontière et plus faciles d'accès, comme Trieste ou Gorizia. Ce rejet du slovène par certains locuteurs transparaît également dans la polémique ${ }^{6}$ autour de la Loi 38/2001 de la région Frioul-Vénétie Julienne ${ }^{7}$ concernant la protection de la minorité slovène, qui a fait craindre à une partie de la population locale la perte de leur propre identité. En Slovénie, en revanche, le résian est bel et bien considéré comme une variété transfrontalière de la langue slovène (cf. Čavdek et al., 2018). Le premier auteur qui, sans réserve et sur une base scientifique solide, reconnaît le résian comme l'un des dialectes qui appartiennent au système linguistique slovène est Fran Ramovš, dans la première moitié du $\mathrm{XX}^{\text {ème }}$ siècle. On a là une belle illustration de l'opposition entre le plan émique et le plan étique, selon les termes proposés jadis par Kenneth Pike (1993) : le premier est celui des représentations, notamment endogènes (ce à quoi l'on croit, ce que l'on pense pertinent et vrai in situ, par conviction ou par tradition), tandis que le second est celui du constat exogène (ce que l'on constate ou que l'on peut décrire avec des outils objectifs, comme la linguistique structurale ; la « vision de l'expert »). Cette dichotomie, inspirée de la paire conceptuelle qui oppose en linguistique l'approche phonémique - qui décrit les catégories abstraites, de manière réductionniste - à l'approche phonétique - qui décrit les réalisations de la parole, de manière atomiste -, revient également à l'opposition entre approche subjective et approche objective, autrement dit, par les catégories et par les vues de l'esprit versus les données sensibles et empiriques.

\footnotetext{
5 [Che simili credenze potessero affascinare in epoche passate non desta alcuno stupore, ma l'aspetto più interessante risiede nel fatto che ancora oggi vengono abbracciate le stesse idee per affermare un'identità e per respingerne un'altra, quella slovena. La maggior parte della popolazione resiana infatti rifiuta l'appartenenza etnica al popolo sloveno dal quale storicamente è sempre rimasta, eccetto brevi periodi, divisa da un confine più o meno impenetrabile.] 6 On peut s'en rendre compte en lisant les conclusions du Conseil Communal de 2010, qui montre que les mêmes débats ont marqué toute la première décennie du XXI ${ }^{\text {ème }}$ siècle : http://www.comune.resia.ud.it/fileadmin/user_resia/ Delibere_Consiglio/2010/DC038-2010.pdf. Les conseillers ont demandé à cette occasion la « sortie » de la Loi $38 / 2001$ en tant que minorité slovène et l'inscription explicite du résian dans ladite loi. Cf. également Toso (2008).

7 Cf/ https://www.gazzettaufficiale.it/eli/id/2001/03/08/001G0093/sg. La loi mentionne explicitement les Slovènes présents dans les provinces de Trieste, de Gorizia et d'Udine.
} 


\section{2 VISIBILITÉ DU RÉSIAN}

Un visiteur potentiel, surtout s'il est porté vers la culture, aborde la découverte du territoire qu'il souhaite visiter par le site communal. La page Internet de la vallée de Resia ${ }^{8}$ se présente comme bilingue : italien / résian et non pas italien / slovène - c'est là une première surprise, pour qui ne serait pas au fait de ce dilemme de la généalogie du résian. Cette information, qui peut sembler aux néophytes une simple curiosité, en dit long aux sociolinguistes que nous sommes : la commune a visiblement cherché à faire de la variété linguistique un outil à part entière, ne serait-ce qu'en partie fonctionnel. Le même site propose en téléchargement libre les numéros de la revue Il giornale di Resia depuis 2009.

Concernant la vie culturelle et les activités qui sont menées pour et en variété locale, une autre page Internet apporte une source d'informations très utiles. Il s'agit du site consacré à la culture et aux traditions : Rezija / Resia ${ }^{9}$, qui regroupe les pages du Cercle culturel résian « Rozajanski Dum », du groupe folklorique « Val Resia », de la chorale « Rože Majave » et de l'association culturelle réunie autour du Musée des habitants du Val Resia. Le site est quadrilingue (italien, slovène, allemand et anglais). A la différence du site communal, ici c'est de la langue slovène qu'il est question, mais c'est également le dialecte qui est utilisé à l'affichage.

Le Cercle culturel résian existe depuis 1983. On lui doit les actions en vue de l'enseignement de la variété locale à l'école, l'organisation de manifestations culturelles mais aussi une intense activité de publications (livres ou journal Näš glas / La nostra voce). Il est à l'origine également d' un colloque organisé en 2008, «Langues et tourisme », consacré précisément aux variétés locales des langues minoritaires comme facteurs d'attraction touristique. Dans sa contribution, Sandro Quaglia insiste sur les changements de comportement des visiteurs après l'ouverture de la vallée aux touristes : "C'est précisément l'élément linguistique qui intéresse les visiteurs qui recherchent constamment des publications en dialecte, des $\mathrm{CD}$ de musique et de chants traditionnels, de divers matériaux qui décrivent la culture résianne. $»^{10}(2014: 69)$.

Quant à l'association culturelle réunie autour du Musée des habitants du Val Resia, elle existe depuis 1995. Le musée comporte une section dédiée à la tradition narrative orale, à travers les contes, les récits, les fables, les légendes et les chants populaires, souvent sous forme bilingue avec l'italien. Par ailleurs, il existe un guichet linguistique

8 Cf. http://www.comune.resia.ud.it/.

9 Cf. https://rezija.com/it. Le site est riche en informations sur la variété linguistique locale dont on apprend la survie dans un contexte de forte diglossie avec l'italien et d'une distanciation (due notamment à un développement séparé, en contexte d'isolement) avec le slovène. Les gens la parlent peu, mais une (faible) présence est assurée à l'école. La langue est audible à la radio (notamment à travers l'émission « Te rozajanski glas » sur Rai di Trieste) ou visible dans la presse (ex. Näs̆ glas / La nostra voce). La musique, la danse ou la tradition orale y sont également mises à l'honneur.

10 [È proprio l'elemento lingua ad interessare i visitatori che sono alla ricerca costante delle pubblicazioni in dialetto, dei CD di musica e canti tradizionali, di vario materiale che descriva la cultura resiana.] 
à Stolvizza auprès de la Bibliothèque communale ${ }^{11}$. On peut mentionner également quelques autres sites qui ne sont pas dénués d'intérêt, comme celui consacré à la variété locale : valresia.it ${ }^{12}$, le site sur les nouvelles du Val Resia : Resia News ${ }^{13}$, et le site qui comporte un dictionnaire, mais aussi les textes sur et en résian : Resianica ${ }^{14}$.

A première vue, la population minoritaire, malgré son faible poids démographique, se caractérise par une forte identité culturelle. La signalisation bilingue est très présente dans la vallée, comme le montrent les photos suivantes :

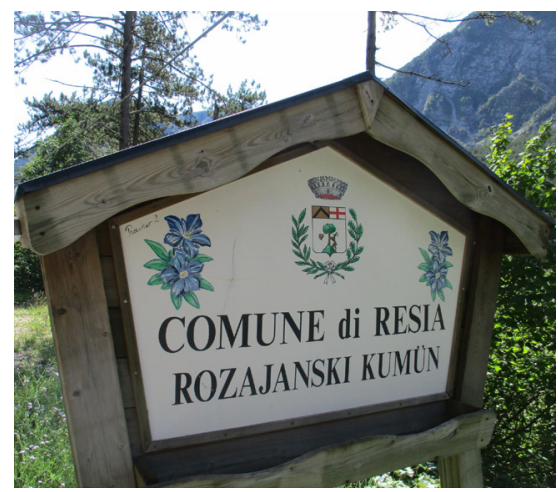

Entrée du Val Resia

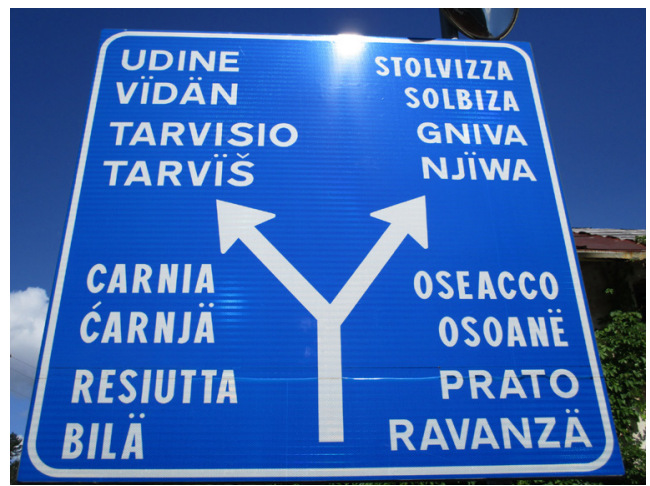

Panneau de signalisation bilingue

11 Au moment même de la rédaction de ce texte (juillet 2021), le guichet a publié une annonce selon laquelle il cherchait un employé capable d'écrire, de produire des textes, de traduire, de promouvoir le résian, donc une personne avec une bonne compétence à l'oral et à l'écrit. L'annonce comporte la question sur la connaissance de l'anglais, de l'allemand, du slovène et d'autres langues. Cf. http://www.comune.resia.ud.it/fileadmin/user_resia/img/ domanda_-_addetto_sportello_linguistico.pdf.

12 Cf. http://www.valresia.it. Le site créé par Sergio Chinese ne semble plus actif, mais il comprend quelques pages qui permettent de comprendre les débats qui ont marqué l'opinion publique autour de la question « dialecte slovène / langue à part ». Dans une lettre ouverte aux 50 scientifiques, en 2008, Sergio Chinese écrit : « Je suis un autodidacte avec peu de connaissances en linguistique, mais la détermination qui anime mon engagement m'est dictée par le cœur et par l'appartenance à un peuple qui voit s'épaissir des nuages menaçants au-dessus de la survie de sa propre identité culturelle. » [Sono un autodidatta con scarse nozioni in materia linguistica, ma la determinatezza che anima il mio impegno è dettata dal cuore e dalla mia appartenenza ad un popolo che vede addensarsi minacciose nubi sulla sopravvivenza della propria identità culturale.]. Cf. http://www.valresia.it/identita/letterastudiosi.pdf. Le site comporte aussi quelques textes en résian.

$13 \mathrm{Cf}$. http://www.resianet.org. Le site possède un onglet sur le résian où l'on peut lire sous la plume du linguiste Han Steenwijk « Si Resia appartient clairement à l'espace slovène de par son parler et sa tradition populaire, les évolutions historiques et sociales à partir de la Renaissance ont créé une situation dans laquelle les Résians ont du mal à s'identifier à la culture slovène, en effet, ils se considèrent comme une population très différente avec sa propre langue et sa propre culture. » [Se per il suo parlare e per la sua tradizione popolare Resia appartiene chiaramente all'area slovena gli sviluppi storici e sociali dal Rinascimento in poi hanno creato una situazione in cui i resiani stentano a identificarsi con la cultura slovena, anzi, si vedono come una popolazione ben diversa con una propria lingua e cultura.]. On doit au même auteur néerlandais Han Steenwijk les récents manuels de référence pour la codification et la standardisation polynomique du resiano (1994a-b). Son autorité est d'autant plus reconnue, outre ses compétences de linguiste, que cet expert vient de l'extérieur, garantissant le prérequis de neutralité glottopolitique.

14 Cf. http://147.162.119.1:8081/resianica/docs/home.jsp. Le linguiste Han Steenwijk, avec quelques collaborateurs, est à l'origine de ce site. Les ressources disponibles avoisinent une cinquantaine d'articles, une vingtaine de textes et un dictionnaire avec 2761 entrées. 


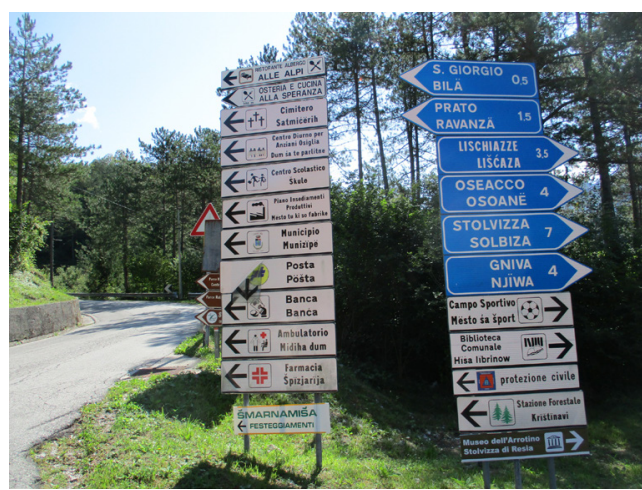

Signalisation bilingue, routière et urbaine

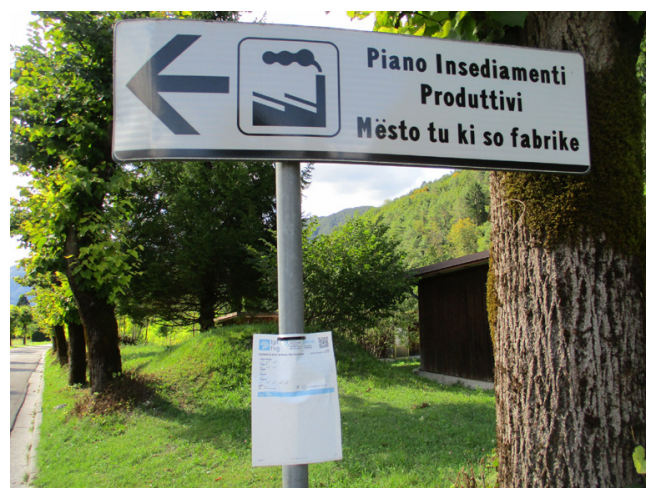

Signalisation bilingue, zone industrielle

Comme le montrent les photos ci-dessus, le paysage linguistique rend compte du bilinguisme de la vallée, dans la variété locale de slovène. On peut y voir également l'implémentation de la codification, élaborée depuis les années 1990, par un patient travail qui a porté ses fruits, avec reconnaissance officielle par la municipalité.

\section{3}

\section{EN FEUILLETANT LA PRESSE...}

Pour terminer ce panorama sociolinguistique à partir de notre regard extérieur, il nous reste à envisager ce qui s'écrit dans la presse locale, concernant les questions linguistiques. Pour ce faire, nous avons feuilleté l'hebdomadaire Novi Matajur, principal organe d'information pour les Slovènes de la province d'Udine, sans être exclusivement réservé à la vallée de Resia ${ }^{15}$. Le journal s'inscrit dans une longue tradition - le premier numéro est paru en 1950 - et la diversité dialectale y est à l'honneur. Nous avons procédé par l'association des mots-clés « Resia + lingua $»^{16}$. Sans surprise, la « russité » présumée des Résians a trouvé un certain écho dans la presse. L'article « Resia n'est pas "un village russe d'Italie" $\rangle^{17}(N M, 15 / 06 / 2016)$ rend compte de la visite d'une délégation russe invitée dans le cadre d'un projet de jumelage sur la base des prétendus liens linguistiques entre le résian et le russe, en précisant : « Ce n'est pas la première fois que nous sommes confrontés à des revendications similaires, mais à ce niveau-là, elles suscitent une certaine perplexité, compte tenu de la réalité des faits qui les contredisent $\gg^{18}-$ bel exemple d'opposition entre l'approche émique locale - pour certains locuteurs - et l'approche étique extérieure - notamment des linguistes. On retrouve les points de

\footnotetext{
15 Cf. https://novimatajur.it/.

16 La langue d'entrée dans le corpus a été pour nous ici l'italien.

17 [« Resia non è un "paese russo d'Italia" »].

18 [Non è la prima volta che ci troviamo davanti a simili affermazioni, ma a questi livelli destano qualche perplessità considerata la realtà dei fatti, che non è proprio così.]
} 
vue opposés également dans l'article «Roberti rencontre Chinese et promet une nouvelle étude "linguistico-culturelle" sur le résian. Qui reste un dialecte slovène »" ${ }^{19}(N M$, 21/03/2019). Le conseiller régional y affronte le maire de l'époque, fervent défenseur du caractère non-slovène du résian, en confrontant les études produites par des chercheurs, notamment linguistes, aux décisions prises par les hommes politiques, non spécialistes. En 2019, dans l'article « Un bien précieux à conserver » ${ }^{20}(N M, 08 / 11 / 2019)$, on lit :

Dimanche j'ai ressenti, dans de nombreux discours, une prise de conscience forte : le résian est un bien précieux qu'il faut préserver. Cela doit être la priorité pour chacune de nos variantes dialectales. Puis, bien plus tard, viennent les discussions. Pourquoi pas, mais on doit toujours tenir compte de ce qui est une évidence : chacun est libre d'avoir sa propre identité, mais ne peut pas nier ce que les savants en langues disent et répètent depuis très longtemps. ${ }^{21}$

On comprend en lisant les différents articles que ce sont finalement là deux logiques qui s'opposent : celle de certains locuteurs traditionnels et celle des chercheurs, les premiers étant parfois instrumentalisés pour des raisons politiques, y compris dans les vallées voisines.

Ce n'est pas la première tentative, et ce ne sera pas la dernière, d'essayer d'enlever à la langue des vallées de Natisone et de Benecia en général, sans parler de Resia, sa principale caractéristique, cet adjectif "slovène" que la politique, l'idéologie et l'ignorance ont trop souvent dénigré et essayé de cacher. $»^{22}$ (« 'Écritures', l'évolution des alphabets et le point sensible du dialecte slovène $\left.»^{23}, 08 / 11 / 2018\right)$.

La question de l'alphabet utilisé pour le résian (ce qui correspond à la dimension de la codification pour les sociolinguistes), sur lequel porte l'article que nous venons de citer, a été également à l'ordre du jour du colloque consacré à la langue slovène qui s'est tenu à Gorizia («Conférence sur la minorité linguistique slovène, solutions partagées » 24 , 29/11/2017), car là aussi, deux modèles s'opposent :

19 [« Roberti incontra Chinese e promette un nuovo studio "linguistico-culturale" sul Resiano. Che resta un dialetto sloveno $"]$

20 [« Un bene prezioso da conservare »].

21 [Domenica ho sentito, nei molti interventi, una forte consapevolezza: il resiano è un bene prezioso che va conservato. Questa deve essere la priorità per ognuna delle nostre varianti dialettali. Poi, molto dopo, vengono le disquisizioni. Che ci possono essere, ma che devono sempre tenere conto di quella che è un'evidenza: ognuno è libero di avere una propria identità, ma non di negare quanto gli studiosi delle lingue ci dicono e ripetono da tantissimo tempo.] 22 [Non è il primo tentativo, e non sarà l'ultimo, di cercare di togliere alla lingua delle Valli del Natisone e della Benecia in generale, per non parlare di Resia, la sua caratteristica principale, quell'aggettivo 'slovena' che politica, ideologia ed ignoranza hanno troppe volte denigrato e cercato di nascondere.]

23 [« 'Scritture', l'evoluzione degli alfabeti e il tasto dolente del dialetto sloveno »].

24 [« Conferenza sulla minoranza linguistica slovena, le soluzioni condivise »]. 
[...] un [modèle] "académique" (élaboré par le slavisant Han Steenwijk) et un autre rendu officiel par une résolution du conseil municipal. Pour une solution partagée, dans le document final du colloque, avec l'accord de toutes les parties, la Région est tenue de convoquer une réunion d'experts sur le sujet susceptible de résoudre le problème en adoptant une orthographe unitaire et reconnue. ${ }^{25}$

Cette voie conciliante n'est pas inaudible, et le slovène standard et le résian peuvent tout à fait partager le même espace, selon certains responsables locaux, comme par exemple Luigia Negro, de l'Union régionale économique slovène ("Luigia Negro (URES) : "Notre communauté slovène face à de nouveaux défis" " $\left.{ }^{26}, 02 / 12 / 2015\right)$.

Le thème le plus significatif, je dirais, concerne l'enseignement bilingue dans notre province. J'ai toujours aimé étudier les langues et je trouve donc naturel que le slovène soit enseigné dans le coin, en tenant compte des variations locales bien sûr, à partir desquelles on pourrait partir. Nos aînés connaissaient plus que quelques langues. ${ }^{27}$

Ainsi donc le principal organe de presse régional pour la minorité slovène se fait écho des débats qui ont secoué la société ces dernières années. Il serait également intéressant, dans un travail futur, de compléter cette lecture sociolinguistique de Novi Matajur par celle de deux autres organes de presse, à caractère davantage local : Il giornale di Resia et Näš glas / La nostra voce. En ce qui concerne ce dernier bulletin d'information, qui bénéficie du soutien financier de la Région Friuli Venezia Giulia et de l'Union des Cercles Culturels Slovènes de ladite région, il s'agit de fascicules d'une quinzaine de pages, contenant des articles aussi bien en slovène dialectal que standard et en italien - le choix de la langue dépend des contenus présentés. Chaque numéro est abondamment illustré de photos et de comptes-rendus sur les activités associatives et les visites de responsables politiques, chercheurs ou délégations, ainsi que sur les événements festifs et les inaugurations et commémorations d'institutions culturelles locales ${ }^{28}$. C'est un véritable organe de liaison, à visée pragmatique, qui permet de suivre l'évolution des initiatives locales et

$25[[\ldots]$ una "accademica" (elaborata dallo slavista Han Steenwijk) ed una resa ufficiale da una delibera del consiglio comunale. Per una soluzione condivisa, nel documento finale del tavolo, con l'accordo di tutte le parti, si richiede che la Regione convochi un tavolo di esperti in materia che possa risolvere il problema adottando una grafia unitaria e riconosciuta.]

26 [« Luigia Negro (SKGZ) : "La nostra comunità slovena di fronte a nuove sfide" »].

27 [Il tema più significativo direi che riguardi l'insegnamento bilingue nella nostra provincia. Lo studio delle lingue mi è sempre piaciuto e quindi trovo che sia naturale che dalle nostre parti si insegni lo sloveno, tenendo conto anche delle varianti locali naturalmente, dalle quali si può partire. I nostri vecchi conoscevano più di qualche lingua.]

28 Voir https://rezija.com/it/circolo-culturale-resiano-rozajanski-dum/pubblicazioni/nas-glas-la-nostra-voce/nasglas-la-nostra-voce-2011/ ou, plus récemment https://rezija.com/wp-content/uploads/2021/03/Nas-Glas-La-nostravoce-dec.-2020.jpg, etc. pour accéder à des sommaires et à des fac-similés. 
régionales, les événements et les spectacles liés à la culture et à la langue, ainsi que les liens avec l'étranger (visites de migrants de la diaspora, de chercheurs slovènes, etc.).

\section{4}

\section{REGARD DU SOCIOLINGUISTE}

Dans l'arrière-plan des débats identitaires, politiques et idéologiques, un travail de codification et de normativisation a été mené avec un relatif succès. On pense ici notamment à l'importante contribution de Han Steenwijk, chercheur d'origine hollandaise, mais spécialiste reconnu en Italie de ces questions ${ }^{29}$.

Face au dilemme de la généalogie contestée du dialecte de Resia, entre plan émique et plan étique, nous proposons d'utiliser un modèle d'analyse des situations de contact et de conflit de langues particulièrement apte à traiter la complexité des situations : le $\mathrm{Mo}$ dèle d'Écologie de Pressions ${ }^{30}$. Ce modèle se fonde sur les principes suivants : premièrement, toute situation sociolinguistique constitue un état des choses ou un état du monde local, qui est le produit complexe des interactions entre groupes humains en présence au cours de l'histoire, avec la formation de narrations ou de récits émiques qui découlent des relations de pouvoir entre contrées, pays et groupes humains. Deuxièmement, ce n'est pas la langue ni une langue seulement qui est au centre de toute situation de contact ou de conflits de langues, mais un répertoire de langues et de variétés, constituant une compétence linguistique. Dans le cas du résian et des dialectes de la constellation géolinguistique slovène locale (ex. tersko et nediško), les composantes du répertoire ont été de longue date, outre le dialecte slovène, le frioulan d'abord, l'italien ensuite, davantage que le slovène - lequel a connu une évolution glottopolitique le conduisant à une individuation par la standardisation de l'autre côté de l'actuelle frontière. Le répertoire des slovénophones de Slovénie a, de son côté, davantage dû composer avec des langues comme l'allemand (hégémonie autrichienne ou austro-hongroise), puis le serbo-croate. Les deux répertoires ont évolué parallèlement, de manière quasiment cloisonnée, durant des siècles, ce qui a fait « dériver » leurs clés d'interprétation émiques sur leurs généalogies respectives. En outre, sur le plan structural (étique), la nature intriquée, complexe, des répertoires, par le feuilletage des langues et des variétés dialectales, chez les locuteurs des deux groupes, d'un côté à l'autre de la frontière, avec les conséquences émiques que l'on vient de voir (sentiment de non appartenance mutuelle), fait que la compétence linguistique se caractérise, sur le plan fonctionnel des pratiques langagières, par une dimension d'une importance capitale, décisive, appelée facilité partagée. C'est ce qu'on résume communément par la concession « l'important, c'est de se comprendre », qui

29 On lui doit le travail sur l'orthographe et la grammaire du résian dans les années 1990, publié à Padoue.

30 Cf. Terborg et García-Landa (2013), Trujillo Tamez et Terborg (2009). Voir aussi, pour l'ancrage épistémologique de ce modèle dans le cadre de la Théorie de la Complexité : Bastardas i Boada (2013), Bastardas i Boada \& Massip Bonet (2013). 
implique une certaine dose de coopérativité, mais aussi d'habitude, d'usage et de pratique - comme n'importe quel entraînement physique ou mental. Or, cette faculté est acquise en fonction du contexte, de l'état des choses, notamment du bain linguistique, voire du bain sociolinguistique et dialectal. Ainsi, il existe une facilité partagée du point de vue de tout locuteur du slovène standard pour comprendre d'autres variétés dialectales du slovène, tout comme il y a une facilité partagée pour tout locuteur de résian, du moins peuton le supposer, de comprendre des langues romanes (donc génétiquement non proches) comme le frioulan, sur le plan dialectal - y compris ses dialectes, pourtant nombreux -, le dialecte veneto trentinois ou de la région de Venise et, bien entendu, l'italien, comme langue nationale de référence. L'inverse n'est pas vrai : le locuteur slovénophone ne peut guère accéder même à une compréhension passive de l'italien, et encore moins du frioulan, sans un apprentissage spécifique - car il n'est pas plongé dans le bain dialectal des langues rhéto-romanes ou italo-romanes, qui ne font pas partie de son état du monde immédiat. En revanche, le bain linguistique des locuteurs slovènes, en termes de langues slaves, est très diversifié : outre le serbo-croate, les Slovènes ont eu l'occasion d'entendre ou d'étudier de nombreuses autres langues slaves (russe, notamment), et savent aisément distinguer, ne serait-ce qu'intuitivement, entre langues slaves « balkaniques » (par ex. celles en usage dans l'ex-Yougoslavie) et d'ailleurs. Il faudrait même parler de bassins de langues disponibles pour la comparaison, dans l'état du monde cognitif de ces différentes populations : en Italie, un vaste bassin de variétés rhéto-romanes (frioulan, ladin dolomitain) et italo-romanes (veneto, italien régional et standard) ; en Slovénie, un vaste bassin de langues slaves.

Nous avons donc ici deux facteurs relativement statiques, d'une très grande force de gravité : 1) l'état des choses historique, ou les configurations sociolinguistiques locales, régionales et nationales d'une part, et de l'autre, la compétence de communication, notamment du point de vue de sa facilité partagée - celle qui délie les langues, favorise l'échange spontané et surtout, le choix des langues de conversation ou de communication, à l'oral comme à l'écrit. À ces facteurs prégnants s'ajoute une cascade de facteurs dynamiques, car aucune société n'est statique en soi : la société ne reste jamais inerte. Elle ne cesse de s'adapter aux changements internes et externes, aussi lents ou rapides soient-ils, même si toute sa sphère mentale est conditionnée par les deux facteurs statiques que nous venons de mentionner. Ces facteurs dynamiques sont les actions, qui sont entreprises en fonction de divers régimes de praxis (communicative, éducative, etc.) : les intérêts, les besoins, les idéologies, les croyances et les systèmes de valeur - qui correspondent, grosso modo, aux dimensions pragmatique, cognitive et éthique. Chacune de ces dimensions s'entrelace avec les autres, interagit avec les autres, et peut par conséquent fluctuer : les intérêts répondent aux besoins matériels (économiques, sociaux), de même que les idéologies déterminent en partie (mais pas non plus complètement) les croyances - et vice-versa -, qui se nourrissent des systèmes de valeurs. Nous avons vu que le dilemme de l'auto-identification paradoxale des locuteurs de resiano s'explique aisément par l'état 
des choses et par la nature des répertoires sociolinguistiques fondant leur compétence active et passive des langues.

On peut pousser plus loin la réflexion, et chercher à comprendre comment les facteurs dynamiques que nous venons d'évoquer peuvent expliquer ce dilemme. Accepter cette interdépendance, qui favorise le slovène, en tant que variété standard, ne pourrait, à terme, que diriger l'action en faveur de la langue locale vers... sa substitution par la variété « légitime ", standard, du pays limitrophe - on voit, par exemple, le croate standard enseigné dans les écoles de l'aire croatophone molisane, alors que le dialecte croate molisain est un dialecte ikavien très différencié, qui mériterait d'être renforcé, mais se retrouve marginalisé en milieu scolaire par ce choix de subordination à la variété « légitime ».

Quel besoin y a-t-il, de reconnaître l'affiliation étique au slovène et à son réseau dialectal, à son diasystème ? Certes, on peut en attendre un bénéfice économique : par ses caractéristiques originales, le resiano est une pièce du puzzle de la complexité de la langue slovène et de son histoire, et qu'on le considère comme représentation d'un état structural ancien, pré-standardisation du slovène, ou comme une variété fortement innovante, la singularité de cet état de langue a de quoi attirer les touristes slovénophones. C'est le facteur « langue-pont» ou « dialecte-pont », à échelle transfrontalière. Sur le régime idéologique, les motivations et l'arrière-plan historique est complexe : deux idéologies nationales s'affrontent alors, et la dimension hégémonique s'avère inévitable. La diversité linguistique et dialectale en Italie a connu des périodes difficiles, voire sombres, par le passé - la politique centralisatrice, assimilationniste de Benito Mussolini a laissé des traces dans la société civile, et loin de l'image d'Epinal que l'on peut se faire d'un Eldorado de la diversité linguistique, l'Italie doit la survie de cette diversité à une farouche résistance et une forte résilience des répertoires multilingues et multidialectaux. La même période fut marquée par les questions d'irrédentisme, et des conflits interfrontaliers plus ou moins larvés - c'est là une litote. On peut donc comprendre que, par endroits, une certaine prudence reste de mise. Même dans le cadre d'une Union européenne consensuelle et « confraternelle », les cicatrices du passé peuvent affleurer, dans la conscience collective. Du point de vue des croyances, celle de l'origine russe du resiano en est une, on l'a vu, qui relève du plan émique, mais qui ne cristallise pas moins un enjeu, un objet de négociation et d'action politique entre secteurs de la société locale (avec leurs besoins, intérêts, etc.).

Enfin, en termes de systèmes de valeur, l'attachement au local, à la spécificité irréductible, est d'autant plus compréhensible que le secteur resianophone est moins constitué de néolocuteurs urbains que de locuteurs d'un certain âge, héritiers de la civiltà contadina alpine, qui tient à préserver son territoire et son ancrage territorial, dans un arc alpin qui a connu, ces dernières décennies, un double processus : fort dépeuplement avec exode rural vers les centres urbains des plaines d'une part, repeuplement par des résidents venant d'ailleurs dans le cadre de «l'habitat amène » et de l'économie du tourisme alpin et de la gentrification des zones rurales autrefois périphériques, aujourd'hui plus ou moins 
avantageusement situées dans le deuxième ou troisième cercle de grandes conurbations tressant des « eurorégions $»^{31}$.

En somme, ce que peut apporter la sociolinguistique pour l'analyse d'une situation comme celle-ci, c'est la compréhension du contexte - l'herméneutique, en quelque sorte, d'un état du monde paradoxal, autour de ce dilemme entre vision étique et émique de la nature du resiano. Les deux camps tiendront toujours leurs positions de manière inexpugnable : les partisans de la vision étique ne pourront nier les faits, les observations empiriques, qui ne font que confirmer l'appartenance du resiano, sinon au réseau dialectal slovène (puisque le resiano est situé en dehors de cet espace, en termes de frontières politiques), du moins à son diasystème, sur le plan structural, dans la généalogie et la typologie des langues slaves méridionales; mais nous venons de voir que maints facteurs confortent le point de vue émique des partisans d'une individuation unique, singulière, de cette même variété. Le Modèle d'Écologie de Pressions a des conséquences aisées à comprendre, qui relèvent de la logique triviale des vases communicants : plus l'action des politiques exercera de pressions en faveur de la thèse émique de l'idiosyncrasie irréductible de la langue (ou, par défaut, réductible à la croyance d'une affiliation avec le russe, ou le caractère «non slovène » de la langue), plus les secteurs défendant l'approche étique protesteront et résisteront à cette imposition, en fonction de leur régime de vérité (système de valeurs). En revanche, plus l'action des linguistes suivra la logique d'une affiliation avec le slovène, langue d'un pays voisin, constituée et outillée de longue date en tant que langue standard, littéraire, officielle, plus on pourra s'attendre à un système de conséquences hautement prédictible, tendant à substituer une variété « vernaculaire » par une variété « véhiculaire », toutes choses égales par ailleurs.

Chaque pression exercée à un bout de la chaîne enclenchera son système de conséquences. La pression émique ira vers davantage de distanciation entre resiano et slovène standard (et les variétés du diasystème), davantage de polarisation, mais aussi de fragmentation (vis-à-vis du nediško, mais aussi, bien évidemment, des variétés frontalières de slovène, dans la plaine). C'est un scénario possible, relevant, techniquement, d'une tactique défensive, face au risque d'acculturation par le slovène standard. Il permettrait certes d'élaborer la langue, par distanciation, mais il diminuerait son potentiel de facilité partagée dans les échanges avec les locuteurs de slovène standard ou dialectal, ou avec ceux des variétés slovènes d'Italie. Il amoindrirait, en termes d'intérêt, l'attractivité de cette variété, en tant que langue-pont vers un autre pays de l'UE, mais aussi comme pont vers l'apprentissage des langues slaves (russe y compris, paradoxalement, puisqu'une « convergence privilégiée » avec le russe, sur le plan structural, est une fiction). Un autre scénario consisterait à faire converger l'élaboration fonctionnelle, à l'écrit et dans le domaine audio-visuel, du resiano, avec les dialectes slovènes frontaliers proches et de Haute

31 Cf. l'édifiant article de Steinicke et al. 2012, qui présente le paradoxe des « villages alpins fantômes » vs. les nouveaux semis d'élégants chalets gentrifiés, au gré des vallées alpines frontalières entre Italie et Slovénie, précisément. 
et Basse Carniole, voire avec le slovène standard, favorisant les échanges transfrontaliers et l'apprentissage des langues slaves, ce qui correspond à un besoin réel, dans le cadre de l'UE à 24, dont pas moins de six pays sont de langue slave : Slovénie, Slovaquie, République Tchèque, Pologne, Croatie, Bulgarie. Il conforterait l'attractivité touristique pour les ressortissants de ces pays - mais surtout, certes, pour les Slovènes.

Aucun de ces deux chemins n'est dénué d'embûches, de ressentiment et de conflit potentiels. D'où l'importance de dialoguer, en faisant valoir les divers points de vue et les perspectives, en termes d'intérêts et de besoins ou d'éthique. Ceci dit, la croyance en une singularité du resiano n'est pas non plus dénuée d'intérêt, si elle peut éviter la substitution de la variété locale par la variété standard. Elle a donc son utilité, en tant qu'agent immunitaire, en quelque sorte. L'important, c'est que cette posture émique ne prenne pas un tournant doctrinaire et ne finisse pas sous forme de polémique ou de polarisation empêchant tout dialogue, ou tout réalisme. Mais on pourrait en dire autant de l'option étique, qui deviendrait contreproductive si elle venait à imposer de force une action en faveur de la langue plutôt que des variétés dialectales locales, dans leur intégrité structurale et ethnolinguistique. On voit, une fois de plus, que la langue est à la fois facteur et enjeu de débat démocratique : en soi, elle n'est ni bonne ni mauvaise. C'est la qualité qu'on lui confère, en fonction d'intérêts, de besoins, de croyances, d'idéologies ou de systèmes de valeurs, qui fait basculer la langue dans le vert ou dans le rouge, du point de vue de la rationalité ou de la faisabilité de toute action d'aménagement linguistique.

Enfin, un troisième scénario est possible (quoique ni attesté par le passé, ni observable actuellement) : joindre le pont à la citadelle - à la manière d'un pont-levis -, en favorisant une convergence à la fois de la codification et d'une standardisation du resiano aux côtés des variétés slovènes voisines, mais aussi des variétés proches de la frontière italo-slovène, ce qui supposerait de sérieusement retourner la diglossie. Autant le premier scénario ne ferait que consolider la diglossie fishmanienne envers l'italien, et fergusonienne envers le slovène, en creusant l'isolement et l'antagonisme de fait, tandis que le deuxième scénario risquerait de diluer la diglossie dans un cosmopolitisme assimilationniste, autant le troisième consisterait en une habile et fédératrice forme de normalisation des relations de voisinage et transfrontalières, où les variétés locales resteraient maîtresses du jeu. Une telle option requiert une bonne dose d'intercommunalité, de pluralisme, de solidarité et d'inventivité, mais aussi des compétences de haut niveau, afin de réussir la synthèse. Elle impliquerait aussi une réelle et intense transmission intergénérationnelle de la langue, qui ne semble pas prévaloir dans les faits.

\section{CONCLUSION}

Nous étions loin de penser à tout cela, avec Gregor Perko, quand nous analysions les radicaux thématiques et athématiques de la flexion verbale dans les dialectes slovènes. 
Nous constations que le resiano apportait des données de toute première importance pour la compréhension des mécanismes de différenciation des dialectes slovènes. Mais nous ignorions tout de ces dilemmes ethnolinguistiques, durant son séjour à Montpellier et à Paris, alors que nous planchions sur des tableaux de données flexionnelles slovènes. Ce n'est que bien plus tard, alors que ce compagnon de route nous manquait déjà tant, que Resia est revenu à notre esprit, comme un lieu à connaître, à visiter, conscient que nous n'avions fait qu'effleurer la question de sa position dans le concert des dialectes slovènes lors de notre travail en commun. Gregor Perko avait une réelle passion pour l'activité de recherche - pas seulement pour ses résultats, qui sont toujours contingents, car la recherche vise à établir des connaissances discutables, révisables, falsifiables, en termes popperiens ${ }^{32}$, et non pas des vérités intangibles. Nous avons découvert sur le tard que le resiano nous réservait une surprise de plus : le dilemme de l'action glottopolitique, en termes d'écologie de pressions, qu'on peut envisager depuis son promontoire haut perché. Pont, citadelle ou pont-levis ?

Mais est-ce que le resiano n'a pas déjà donné les preuves qu'il est à la fois pont et pont-levis ? Il est d'ores et déjà un pont, sur le pan étique, en tant que dialecte éminent, auquel des savants de renommée mondiale, comme Baudoin de Courtenay (1875) ${ }^{33}$, ont consacré des études qui ont marqué l'histoire de la slavistique ${ }^{34}$. Il est, de ce point de vue, un dialecte décisif (cf. Alvarez-Pereyre et al. 2013). Par son élaboration par distanciation, par sa singularisation émique, il est une citadelle, et à ce titre, la mise à distance du slovène standard peut être perçue, en termes d'écologie de pressions, comme une action relevant de l'expérience à tenter afin d'éviter l'assimilation à un standard. Il y aurait probablement aussi d' autres raisons qui jouent un rôle en arrière-plan, sur la durée moyenne : les attitudes idéologiques et politiques qui interfèrent aujourd'hui encore dans cette question de la langue slovène dans le Frioul, en raison du passé communiste de la Slovénie, ce qui affaiblit encore le potentiel du troisième scenario esquissé dans cet article.

Nous ignorions tout de cette spécificité, lorsque nous tracions des plans sur la comète avec Gregor Perko pour donner une continuation sur le terrain à nos spéculations théoriques sur les mécanismes de diversification interne du diasystème slovène, dans sa morphologie verbale. Ce fut donc avec bien plus et bien mieux que de la nostalgie que

\footnotetext{
32 Cf. Popper $(1934,1963)$.
}

33 Cf. Mugdan 1984, panorama cependant tempéré par la recension de Stankiewicz (1988), non quant à l'apport de Baudoin de Courtenay en soi, mais plutôt pour la sous-formulation de la synthèse proposée par Mugdan, tant l'apport de ce savant reste complexe, à l'aube de la linguistique moderne.

34 Les données et les hypothèses de divers ordres formulées en son temps par Baudoin de Courtenay ont depuis été revisitées à la lumière des données et des théories modernes, notamment par Steenwijk (1987, 1988). C'est précisément cette dynamique empirique critique de toute entreprise de description linguistique qui constitue la valeur du point de vue étique. Comme le rappelait le bulletin Gazzettino du 27 décembre 1998 (cité par Paletti 2016 : 367), le célèbre linguiste russe d'origine polonaise reconnaissait déjà, en 1872, quatre regroupements linguistiques slaves : résian, Val Torre, Natisone, Castelmonte. Il réfutait que les Résians fussent d'origine russe, même s'il les considérait comme le résultat d'une miscégénation ethnographique slave, avec des éléments d'interférence avec une autre lignée slave (?) - on ne parlait pas encore de contact de langues, à l'époque, mais aujourd'hui on penserait plutôt au contact avec la « lignée » rhéto-romane du frioulan, comme facteur de spécificité structurale, à titre interférentiel. 
nous nous sommes rendus en août 2021 à Resia, puisque nous y avons trouvé confirmation de l'importance du rôle social du linguiste, et des liens fusionnels, organiques, entre la linguistique descriptive et la linguistique appliquée. On peut y déceler une leçon exemplaire, dans notre métier, face aux impératifs qui président à la destinée de nos institutions d'enseignement supérieur - formalisme contre utilitarisme. Autrement dit, la polarité qui oppose tour d'ivoire versus terrain trouve ici une manifestation concrète... de manière analogue à la figure de la citadelle contre le pont, observable dans la complexité des situations sociolinguistiques et glottopolitiques, dans le monde des représentations et des pratiques langagières.

\section{BIBLIOGRAPHIE}

ALVAREZ-PEREYRE, Franck/Sylvie ARCHAIMBAULT/Jean Léo LÉONARD (éd.) (2013) Dialectes décisifs, langues prototypiques, Histoire Epistémologie, Langage, tome 35-1.

BASTARDAS I BOADA, Albert (2013) Complexity and Language Contact: a Socio-Cognitive Framework. S. Mufwene / F. Pellegrino, C. Coupe (ed.) Complexity in Language: Developmental and Evolutionary Perspectives. Cambridge: Cambridge University Press.

BASTARDAS I BOADA, Albert/Àngels MASSIP-BONET (ed.) (2013) Complexity perspectives on language, communication and society. New York: Springer.

BAUDOIN DE COURTENAY, Jan (1875) Opyt fonetiki rez'janskich govorov, Varsava-Peterburg 1895 Materialen zur südslawischen Dialektologie und Ethnographie. I.- Resianische Texte. St. Petersburg.

ČAVDEK, Julian et al. (2018) Mi, Slovenci $v$ Italiji $=$ Noi, sloveni in Italia $=W e$, the Slovenes in Italy. Trst ; Gorica : Slovenska kulturno gospodarska zveza : Svet slovenskih organizacij.

DAPIT, Roberto (2001) Identità resiana fra « mito » e ideologia : gli effetti sulla lingua. Slavica Tergestina 9, 301-319.

LEONARD, Jean Léo/Gregor PERKO (2020) De la Val Resia à la Mura. Esquisse de modélisation diasystémique de la conjugaison slovène par le centre (transitionnel) et par les marges. Verbum XLII 1-2, Modélisation diasystémique, 85-129.

MUGDAN, Joachim (1984) Jan Baudouin de Courtenay (1845-1929) - Leben Werk. Munich: Wilhelm Fink Verlag.

PALETTI, Luigi (ed.) (2016) Pagine di storia. Resoconti di Vita Resiana. Vol. V [19912000], Circulo Culturale Resiano "Rozajanski Dum".

PIKE, Kenneth (1993) Talk, thought and thing: the emic road toward conscious knowledge. Dallas: Summer institute of Linguistics.

POPPER, Karl (1934) Logic der Forschung. Wien : Springer. 
POPPER, Karl (1985) Conjectures et réfutations, La Croissances du savoir scientifique. Paris : Payot.

QUAGLIA, Sandro (2014) Le esperienze di turismo linguistico in Val Resia. Atti del convegno à cura di Luigia Negro Lingue e turismo. Le varianti locali delle lingue di minoranza come elementi di richiamo turistico, 68-71.

STANKIEWICZ, Edward (1988) The Place of J. Baudouin de Courtenay in the History of Modern Linguistics. Russian Linguistics 12-2, 133-145.

STEENWIJK, Han (1994a) Ortografia resiana / Tö jošt rozajanskë pïsanjë. Padova: CLEUP.

STEENWIJK, Han (1994b) Grammatica pratica resiana. Il sostantivo. Padova: CLEUP. STEENWIJK, Han (1988) The fate of the circumflex sign in Baudouin de Courtenay's resian notes. Studies in Slavic and General Linguistics, 11, Dutch Contributions to the Tenth International Congress of Slavists. Linguistics, 495-506.

STEENWIJK, Han (1987) Puzzling evidence: an accented vowel system based on baudouin de courtenay's resian texts. Studies in Slavic and General Linguistics 10, Dutch Studies in South Slavic and Balkan Linguistics, 237-274.

STEINICKE, Ernst/Judith WALDER/Roland LÖFFLER/Michael BEISMANN (2011) Minorités linguistiques autochtones des Alpes italiennes. Revue de Géographie Alpine | Journal of Alpine Research [Online] 99-2 | 2011, http://journals.openedition. org/rga/1469, consulté le 14 juillet 2011.

STEINICKE, Ernst/Peter ČEDE/Roland LÖFFLER (2012) In-migration as a new process in demographic problem areas of the Alps. Ghost towns vs. amenity settlements in the alpine border area between Italy and Slovenia. Erdkunde 66-4, 329-344.

TERBORG, Roland/Laura GARCIA-LANDA (2013) The ecology of pressures: towards a tool to analyze the complex process of language shift and maintenance. A. Bastardas i Boada, À. Massip-Bonet (ed.) Complexity perspectives on language, communication and society. New York: Springer, 219-239.

TOSO, Fiorenzo (2008) Alcuni episodi di applicazione delle norme di tutela delle minoranze linguistiche in Italia. Ladinia XXXII, 165-222.

TRUJILLO TAMEZ Isela/Roland TERBORG (2009) Un análisis de las presiones que causan el desplazamiento o mantenimiento de una lengua indígena de México: El caso de la lengua mixe de Oaxaca. Cuadernos Interculturales, Año 7, 12, 127-140.

POVZETEK

\section{SOCIOLINGVISTIČNA OPREDELITEV REZIJANSKEGA NAREČJA}

Slovensko mikronarečje, značilno za Rezijo in sosednja slovensko govoreča območja v Furlaniji - Julijski krajini, je od slovenskega narečnega območja ločeno z gorsko pregrado. Rezijanščina in 
njej sorodna narečja so že dve stoletji deležni najrazličnejših raziskav, ki prispevajo k obujanju rezijanskega jezika in kulture. Jezikovno in kulturno oživitev rezijanščine omogočajo različne jezikovne strategije, ki ob aktivni podpori kulturnih društev in organizacij potekajo v smeri od spodaj navzgor. V 90. letih 20. stoletja se je začel idiosinkratičen proces normiranja rezijanščine, katerega podlaga so raziskave narečne raznovrstnosti v rezijanski mikroregiji. Pri tem je mogoče opaziti s sociolingvističnega in političnega vidika nasprotujoče si poglede na proces normiranja. Zagovorniki lokalne individualizacije nasprotujejo obravnavi rezijanščine v okviru slovenskih narečij in poudarjanju podobnosti med slovenščino in rezijanščino; po drugi strani skupina zagovornikov različnih vrst polinomske integracije poudarja pomen stičnih točk med rezijanščino in slovenščino. $\mathrm{V}$ prispevku predstavljamo nasprotja med emičnim in etičnim vidikom obravnave problematike, pri čemer se opiramo na različne pisne vire in rezultate terenskega opazovanja. Predstavljamo tri mogoče modele družbenega razvoja ter oživitve rezijanščine in sorodnih narečij v prihodnje: i) trdnjava (izolacionizem, zavračanje slovenščine kot podlage za normiranje rezijanščine), ii) most (integracija, asimilacija rezijanščine s standardno slovenščino) in iii) dvižni most (polinomski pluralizem, ohranjanje strukturnih značilnosti rezijanščine ob priznavanju stičnih točk s slovenščino). Zdi se, da v zadnjih desetletjih prevladuje model dvižnega mostu, čeprav sta druga dva pristopa še vedno skrito prisotna na določenih območjih. Primer rezijanščine je zaradi nenehnega iskanja ravnovesja med izolacijo in asimilacijo še posebej zanimiv za raziskovanje ogroženosti jezika in za sociolingvistične raziskave.

Ključne besede: Rezija, slovenščina, narečje, sociolingvistika, jezikovne strategije

\section{ABSTRACT}

\section{A SOCIOLINGUISTIC LOOK AT RESIANO}

The Slovene dialect of Val Resia and the neighbouring Slovenian-speaking valleys, in Friuli-Venezia Giulia, is embedded behind a mountain buttress which isolates this micro-dialect area from the Slovenian dialect network. A rich tradition of research has documented Resiano and similar varieties for two centuries already, and contributes to the revival of local language and culture, through forms of "bottom-up" language management, involving active and proficient cultural circles and organizations. An idiosyncratic form of corpus planning took shape in the $1990 \mathrm{~s}$, based on specific research work considering the internal dialect diversity of this micro-region. However, various points of view are opposed in the sociolinguistic and political agora between, on the one hand, those who advocate a localist individuation and reject integration of Resian within the framework of Slovenian dialects, denying kinship with Slovenian, and on the other hand, those who are committed to forms of polynomic integration, cultivating links with Slovene as the reference language. Three models of development are proposed, according to an architectural metaphor of this ethnolinguistic Bildung: i) the fortress (denying affiliation to Slovene, and rejecting it as a model 
for corpus planning), ii) the bridge (enhancing close kinship with Slovene, and converging with its status planning), and iii) the drawbridge (enforcing specificity without rejecting affinity with Slovene, and cross-border cooperation with Slovenia). Nowadays, the drawbridge model seems to prevail, although the fortress and bridge trends are still latent locally. The "Resian case", showing constant search of a balance between conflictual standpoints between separation vs. assimilation, happens to be a particularly interesting situation for language endangerment and sociolinguistic studies.

Keywords: Val Resia, Slovenian, dialect, sociolinguistics, language management

\section{RÉSUMÉ}

\section{UN REGARD SOCIOLINGUISTIQUE SUR LE RESIANO}

Les variétés de slovène du Val Resia, en Frioul-Vénétie Julienne, sont enclavées derrière un contrefort montagneux qui les isole du réseau dialectal slovène. Une riche tradition de recherche les documente depuis deux siècles, et connaît un renouveau grâce à l'aménagement linguistique « de par en bas » (associatif). Une codification a pris forme dans les années 1990, fondée sur un travail de recherche tenant compte de la diversité dialectale interne de cette micro-région. Cependant, divers points de vue s'opposent, sur le plan glottopolitique entre d'une part, les tenants d'une individuation localiste contre une intégration dans le concert des dialectes slovènes, et d'autre part, les tenants d'une intégration polynomique et pragmatique, cultivant le lien avec la langue de référence, le slovène, dans une relation de complémentarité. Le présent article rend compte de ce dilemme et des contradictions entre plan émique et plan étique, sur la base d'un état documentaire diversifié et d'observations de terrain. On propose trois « modèles » ou scénarios » de développement social et de revitalisation du résian et des variétés italo-slovènes proches : la citadelle (isolationnisme, avec risque de substitution sociolinguistique), le pont (intégration, avec risque d'assimilation par le slovène standard), le pont-levis (un pluralisme polynomique, avec ouverture au monde slovène et slave, tout en maintenant une spécificité structurale). Des trois options, c'est semble-t-il la troisième qui tend, fort heureusement, à prévaloir depuis plusieurs décennies, bien qu'il faille rester vigilant à maintenir un équilibre entre individuation, isolement et assimilation. C'est là un processus sans fin, dont le résian constitue un cas exemplaire pour la glottopolitique des minorités transfrontalières.

Mots-clés : Val Resia, slovène, dialecte, sociolinguistique, aménagement linguistique 